\title{
Self-Assembled Nanogels Based on Ionic Gelation of Natural Polysaccharides for Drug Delivery
}

\author{
Huimin Wang, Hong Deng, Menghan Gao and Weiqi Zhang* \\ State Key Laboratory of Medical Molecular Biology and Department of Biomedical Engineering, Institute of Basic Medical \\ Sciences Chinese Academy of Medical Sciences, School of Basic Medicine, Peking Union Medical College, Beijing, China
}

\section{OPEN ACCESS}

Edited by:

Lei Liu,

Jiangsu University, China

Reviewed by:

Ning Han,

Beijing University of Chinese

Medicine, China

Marcelo Calderon

Polymat, Spain

*Correspondence:

Weiqi Zhang

zwq@ibms.pumc.edu.cn

Specialty section:

This article was submitted to Biomaterials

a section of the journal Frontiers in Bioengineering and Biotechnology

Received: 30 April 2021

Accepted: 16 June 2021

Published: 16 July 2021

Citation:

Wang $\mathrm{H}$, Deng $\mathrm{H}$, Gao M and Zhang W (2021) Self-Assembled Nanogels Based on Ionic Gelation of Natural Polysaccharides for Drug

Delivery.

Front. Bioeng. Biotechnol. 9:703559. doi: 10.3389/fbioe.2021.703559
The polysaccharides (PS) have been widely used as biomaterials in drug delivery, due to their excellent biocompatibility, ease of functionalization, and intrinsic biological activities. Among the various PS-based biomaterials, the self-assembled PS nanogels (NG) featuring facile preparation are attracting evergrowing interests in various biomedical applications. Specifically, NG derived from the self-assembly of natural PS well maintain both the physicochemical and biological properties of PS while avoiding the chemical modification or alteration of PS structure, representing a potent drug delivery system for various therapeutic agents. In this review, the natural PS, such as chitosan, alginate, and hyaluronan, for self-assembled NG construction and their advantages in the applications of drug delivery have been summarized. The residues, such as amine, carboxyl, and hydroxyl groups, on these PS provide multiple sites for both ionic cross-linking and metal coordination, which greatly contribute to the formation of self-assembled NG as well as the drug loading, thus enabling a wide biomedical application of PS NG, especially for drug delivery. Future developments and considerations in the clinical translation of these self-assembled PS NG have also been discussed.

Keywords: nanogel, drug delivery, polysaccharides, self-assembly, ionic gelation

\section{INTRODUCTION}

Nanogels (NG) are nanoscale hydrogels that are composed of a three-dimensional network of polymers with the ability to adsorb a large amount of water. The NG has the merits of most of the nanoparticles (NP) for the applications of drug delivery, while it also possesses its own beneficial features due to its unique physiochemical properties (Soni et al., 2016; Xu et al., 2017). With a rational design, NG can have high hydrophilicity, tunable size and porosity, deformability, and degradability. The NG with an appropriate size (i.e., $\sim 10-200 \mathrm{~nm}$ ) can mediate the selective tumor accumulation for anticancer drug delivery by taking advantages of the enhanced permeabilization and retention (EPR) effect and the targeting ligands attached on the NG (Kobayashi et al., 2014; Hartshorn et al., 2018; Cuggino et al., 2019). The gelation of hydrophilic polymer generates NG, and meanwhile, the cross-linking degree controls the NG softness. The softness is a key parameter for the interaction of NP with the biological system (Li et al., 2020). For example, NG of different softness demonstrates a varied cellular uptake (Guo et al., 2018; Zhang et al., 2020). The deformation of NG allows it to pass through the pores that are smaller than the hydrodynamic size of NG, which plays an important role in the in vivo circulation and accumulation of NG at the disease site, e.g., tumor (Hendrickson and Lyon, 2010; Anselmo et al., 2015). The porous 
structure of NG provides large spaces for drug loading such as small-molecule drugs, nucleic acids, and proteins (Mauri et al., 2018; Suhail et al., 2019). The release speed of the drugs, a key factor for the drug delivery system, could be tuned by controlling the cross-linking degree of NG. More importantly, NG are generally hydrophilic and can be degraded when degradable or natural polymers are adopted as NG matrix. Among the naturally occurring polymers, polysaccharides (PS) represent one of the most prevalent biomacromolecules to construct NG for drug delivery.

Polysaccharides are biopolymers composed of repeated monosaccharides linked by glycosidic bonds. Different monosaccharides and their glycosidic linkage patterns generate various PS such as chitosan (CS), alginate (ALG), and hyaluronan (HA) (Liu et al., 2008). PS could be digested by various enzymes and recycled when used as biomaterials in the body. Most of the PS demonstrate low toxicity and immunogenicity, which make them appealing for various biomedical applications such as drug delivery (Barclay et al., 2019). In addition, some PS also demonstrate intrinsic biological activities. For example, HA could be specifically recognized by CD44, a receptor overexpressed on many cancer cells. Consequently, the HAbased nanocarriers could be used for CD44-targeted anticancer drug delivery (Spadea et al., 2019; Gao et al., 2021). The cross-linking of PS through chemical reactions, such as UV light radiation and glutaraldehyde treatment, can generate PS NG with good stability (Debele et al., 2016). The chemical modification of PS provides flexible options to construct NG and load cargoes; however, it may not only compromise the biocompatibility but also influence the biological activities of PS. A high degree of chemical modification could retard the degradation of HA and also compromise the CD44-targeted effects (Bhattacharya et al., 2017; Kim et al., 2019). Self-assembled NG based on natural PS would maintain their biological activities and meanwhile provide the physicochemical properties of NG for drug delivery. The functional groups of PS can also mediate different noncovalent interactions. Specifically, the hydroxyl group of PS can mediate the hydrogen bonding while the amine and carboxyl groups can take part in both electrostatic interaction and metal coordination (Giammanco et al., 2015; Debele et al., 2016; Kim et al., 2017). These non-covalent interactions can involve multiple monosaccharide units simultaneously to cause both intermolecular and intramolecular cross-linking of PS, inducing the formation of self-assembled NG. The preparation of self-assembled PS NG requires neither chemical reactions nor a complicated purification process, representing a facile and cost-effective fashion to construct nanocarriers for drug delivery. The common self-assembly of native PS is mainly mediated by the physical cross-linking either through ionic gelation or through metal coordination, which is reversible. Depending on the PS type, the resultant NG can further entrap various drugs through electrostatic interaction, hydrophobic interaction, and $\pi-\pi$ stacking, thus helping solubilize the drugs as well as increase their bioavailability. Furthermore, the non-covalent interactions between the encapsulated cargoes and PS were also believed to stabilize the self-assembled NG (Zhang and Tung, 2017; Cai and Lapitsky, 2020). In this review, we mainly focused on the self-assembly of native PS, such as CS, ALG, and HA (Table 1), and the related NG for drug delivery. The characteristics of these PS and the representative strategies to prepare the self-assembled NG are summarized. Ionic interaction and metal coordination are the main routes employed to initiate the self-assembly of NG. Recent progress of the self-assembled PS NG for drug delivery is introduced with a focus on both the advantages and the challenges to realize the efficient drug delivery. Finally, a perspective for the future development of self-assembled PS NG in translational medicine has been discussed.

\section{SELF-ASSEMBLED CHITOSAN (CS) NG}

Among the self-assembled PS NG, CS is the most prevalent PS utilized to deliver various types of drugs. CS is a positively charged and linear PS consisting of $N$-acetyl-D-glucosamine (i.e., 2-acetylamino-2-deoxy-D-glucose) units linked by $\beta-1,4$ linkages. CS is produced by the deacetylation of chitin, which is the second most abundant PS on earth (Miao et al., 2018; Qu and Luo, 2020). The CS-based products have been widely used in the food and cosmetic industry as well as medicine such as wound dressing (Morin-Crini et al., 2019). In addition, the bioactivities of CS include antibacterial, antifungal, anti-HIV-1, and antioxidant activities (Cheung et al., 2015). When used in drug delivery, the CS-based NP demonstrate good biodegradability, low toxicity, and mucoadhesive ability (Lapitsky, 2014; Swierczewska et al., 2016). The CS shows poor water solubility at neutral $\mathrm{pH}$, while the acidic environment (i.e., $\mathrm{pH}<6.5$ ) and improved deacetylation can help solubilize the CS (Miao et al., 2018). Due to its primary amino groups, CS appears to be positively charged, which confers its excellent ability to bind anionic therapeutics in drug delivery (Debele et al., 2016; Ojeda-Hernandez et al., 2020). The ionic gelation of CS is one of the most attractive methods to formulate the self-assembled CS NG, which does not require chemical reactions.

The positive charges of CS allow it easily to interact with polyanionic molecules to form hydrogels. Tripolyphosphate (TPP) is the most widely used non-covalent cross-linker for CS due to its non-toxic and multivalent properties (Fan et al., 2012; Fischetti et al., 2020). The negative charges of TPP could efficiently interact with amines on CS through electrostatic interaction, which leads to CS cross-linking and thus the formation of NG. The CS/TPP NG was able to withhold anionic drugs through their electrostatic interaction with CS (Figure 1A). First, Calvo et al. demonstrated that the CS together with polyethylene oxide copolymer could be physically cross-linked by TPP to form NG ranging from 200 to $1,000 \mathrm{~nm}$ to load bovine serum albumin (Calvo et al., 1997). Currently, the typical preparation of drug-loaded CS/TPP NG only requires the mixing of CS, TPP, and the drug of interest followed by a purification procedure. During the self-assembly process, the parameters, such as the $\mathrm{pH}$, temperature, concentrations of TPP and drugs, and the molecular weights of CS, are the key factors to control the physicochemical properties of the CS/TPP NG (Fan et al., 2012; Desai, 2016; Sreekumar et al., 2018). Due to the facile preparation, 
TABLE 1 | Key features of polysaccharides discussed in this review.

\begin{tabular}{|c|c|c|c|c|}
\hline PS & Molecular structure & Sugar units & $\begin{array}{l}\text { Representative biomedical } \\
\text { applications }\end{array}$ & $\begin{array}{l}\text { Biological activity(s) for drug } \\
\text { delivery }\end{array}$ \\
\hline CS & & $\begin{array}{l}\text { D-glucosamine, } \\
\text { N-acetyl-D-glucosamine }\end{array}$ & $\begin{array}{l}\text { Cosmetics, wound dressing, } \\
\text { hemostatic agent, etc. }\end{array}$ & $\begin{array}{l}\text { Encapsulation of anionic drug, } \\
\text { mediating endosomal escape of } \\
\text { drugs }\end{array}$ \\
\hline ALG & & $\begin{array}{l}\text { D-mannuronic acid, L-guluronic } \\
\text { acid }\end{array}$ & $\begin{array}{l}\text { Food additive, dental } \\
\text { impressions, wound dressing, } \\
\text { etc. }\end{array}$ & Encapsulation of cationic drugs \\
\hline $\mathrm{HA}$ & & $\begin{array}{l}\text { D-glucuronic acid, } \\
\text { N-acetyl-D-glucosamine }\end{array}$ & $\begin{array}{l}\text { Dermal fillers, joint lubrication, } \\
\text { preventing postoperative } \\
\text { adhesions, eye drops, etc. }\end{array}$ & $\begin{array}{l}\text { Intrinsic targeting effect for } \mathrm{HA} \\
\text { receptors, e.g., CD44 }\end{array}$ \\
\hline
\end{tabular}

PS, polysaccharides; CS, chitosan; ALG, alginate; HA, hyaluronan.

a quick survey of self-assembly conditions for CS/TPP NG as well as the large-scale manufacturing is possible. An increasing number of anionic drugs, such as small-molecule drugs, nucleic acids, and proteins, have been successfully encapsulated in CS/TPP NG to realize various therapeutic applications (Nogueira et al., 2013; Desai, 2016; Jamil et al., 2016). Recently, Abbaszadeh et al. reported that the CS/TPP NG loaded with quercetin could potentially function as both antibiotic and anticancer agents (Abbaszadeh et al., 2020). When compared with the smallmolecule drugs, the delivery of nucleic acids, such as genes, messenger RNA (mRNAs), and small interfering RNA (siRNAs), faces more challenges due to their vulnerability to nuclease degradation and poor capability to cross the cell membrane. Besides, the endosomal escape of nucleic acids was necessary to ensure an effective transfection, since nuclear and cytoplasmic locations are essential for gene and siRNA delivery, respectively (Degors et al., 2019). The positively charged CS could efficiently bind and compact the nucleic acids, thus protecting them from nuclease degradation. Katas et al. demonstrated that, compared with the direct complexation of CS and siRNA, the CS/TPP NG mediated a better RNA interference (RNAi) effect that was presumably due to the improved binding and loading of siRNA in the NG (Katas and Alpar, 2006). On the cellular uptake of CS/TPP NG, CS can also mediate the endosomal escape of the nucleic acids, since amines of CS induce the proton sponge effect to mediate endosomal destabilization (Nasti et al., 2009; Richard et al., 2013). Similar to nucleic acid delivery, CS/TPP NG could load different proteins, protect them from enzyme degradation, and realize their cytosol delivery (Xu and Du, 2003; Renu and Renukaradhya, 2020). Although the cationic property of CS/TPP provides a flexible delivery platform for different types of drugs, it also brings unnecessary effects. In the case of protein delivery, the strong positive charges of CS may induce the conformational change of protein, which inevitably compromise the protein activity (Bekale et al., 2015; Moraru et al., 2020). Additionally, positively charged CS/TPP NG may damage the cell membrane, causing unexpected cell toxicity (Bowman and Leong, 2006). Similar to other cationic NP, CS/TPP NG also induce the nonspecific adsorption of proteins from the biological fluid, which may not only change the size of NP but also affect the in vivo circulation (Corbo et al., 2016; Moraru et al., 2020). To reduce the positive charges of CS/TPP NG, other anionic polymers could be co-encapsulated to neutralize a part of the positive charges, thus potentially reducing the non-specific side effects.

\section{SELF-ASSEMBLED ALGINATE (ALG) NG}

ALG is the second most used PS to construct ionic hydrogels for different biomedical purposes. ALG is a linear anionic PS mainly derived from brown algae and bacteria, consisting of $\beta$ D-mannuronic acid (i.e., $M$ units) and $\alpha$-L-guluronic acid (i.e., G units) (Debele et al., 2016; Miao et al., 2018). ALG has been approved as food additives and widely used in biomaterials researches ascribing to its minimal toxicity, low cost, ease to formulate hydrogels, and mechanical flexibility (Lee and Mooney, 2012). The functional groups of ALG, such as hydroxyl and carboxyl groups, can non-covalently interact with divalent cations, such as $\mathrm{Ca}^{2+}, \mathrm{Zn}^{2+}$, and $\mathrm{Mn}^{2+}$ (Russo et al., 2007; Brus et al., 2017), which mediate the ionic gelation and the NG formation. The G residues of ALG show a high affinity to divalent cations such as $\mathrm{Ca}^{2+}$ than $\mathrm{M}$ residues. Consequently, ALG with more G blocks leads to hydrogels of better stability compared with ALG that is rich in M blocks (Debele et al., 2016). Nevertheless, the self-assembly of ALG mediated by the electrostatic interaction with cations enables a facile preparation of drug-loaded NG. The resultant NG features the flexibility to deliver different drugs such as chemotherapeutics for cancer, insulin for diabetes, and antibiotics for infection diseases (Severino et al., 2019). 


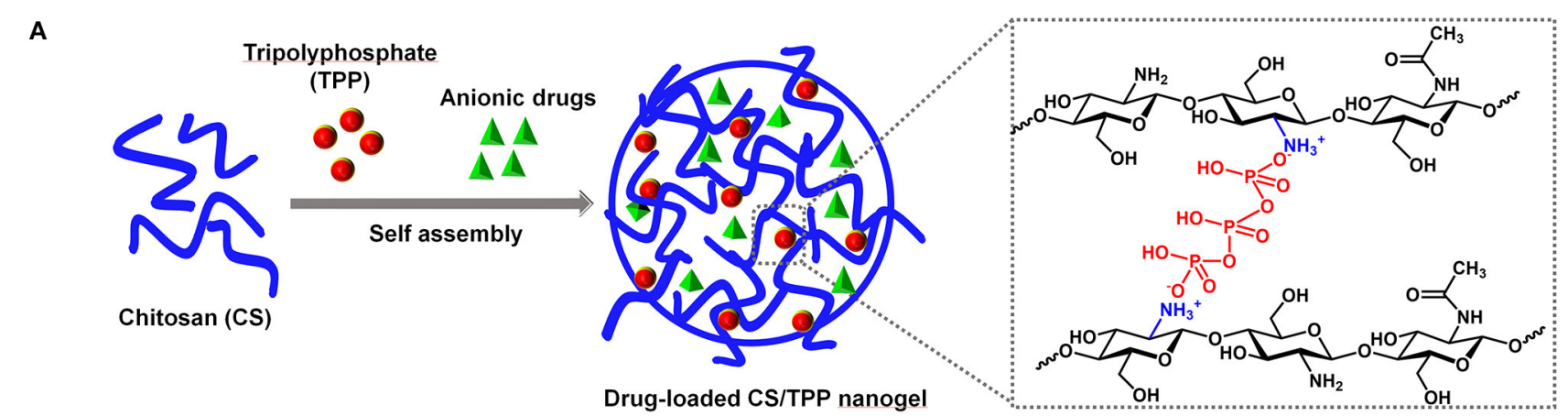

B

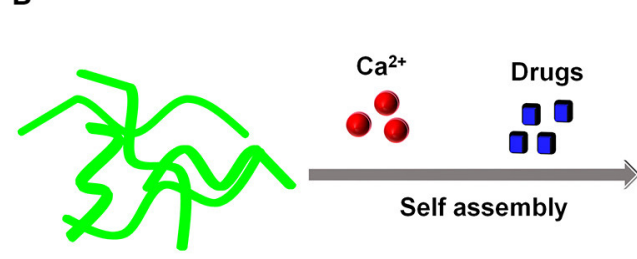

Alginate (ALG)

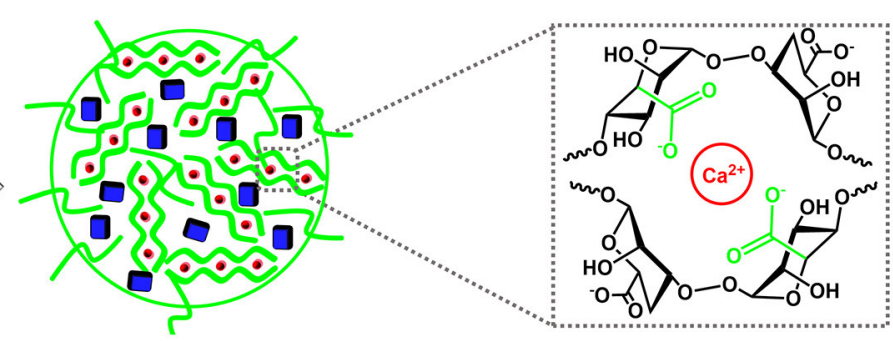

Drug-loaded ALG/Ca ${ }^{2+}$ nanogel

C

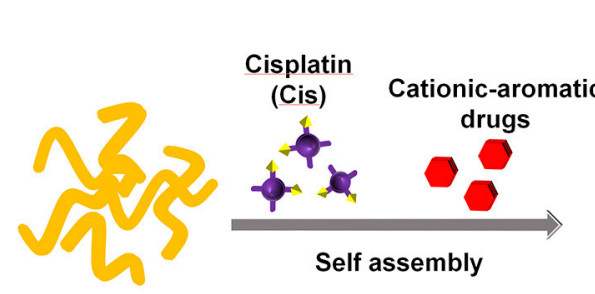

Hyaluronan (HA)

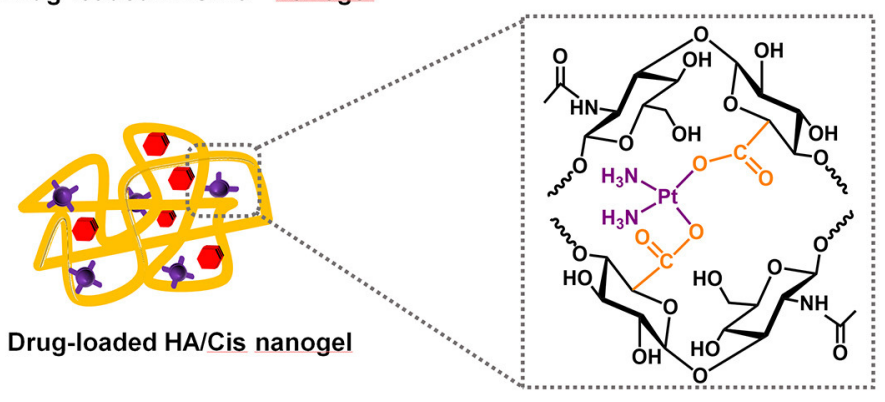

FIGURE 1 | The representative self-assembled nanogels based on the natural polysaccharides (PS) for drug delivery. The order of mixing PS, drugs, and ionic cross-linkers could be varied during the self-assembly process. (A) CS/TPP nanogel, (B) ALG/Ca ${ }^{2+}$ nanogel, (C) HA/Cis nanogel.

Calcium is the most extensively used cation for ALG NG preparation since it is an essential element for the body and easily accessible. First, Grant et al. reported that the ionic interaction between $\mathrm{G}$ blocks and $\mathrm{Ca}^{2+}$ formed an "egg-box" structure and contributed to the physical cross-linking of ALG (Grant et al., 1973; Hu et al., 2021). The $\mathrm{Ca}^{2+}$-mediated gelation could initiate the formation of $\mathrm{ALG} / \mathrm{Ca}^{2+} \mathrm{NG}$ to encapsulate different drugs through self-assembly (Figure 1B). Under the selected preparation conditions, a direct mixing of ALG and $\mathrm{Ca}^{2+}$ could generate drug-loaded NG without the involvement of solvents (Xue et al., 2015; Bazban-Shotorbani et al., 2016). Xue et al. demonstrated that doxorubicin could be electrostatically self-assembled into the $\mathrm{ALG} / \mathrm{Ca}^{2+}$, and thus the NG showed a $\mathrm{pH}$-responsive drug release behavior, excellent compatibility, and anticancer effect (Xue et al., 2015). Generally the ALG/Ca ${ }^{2+} \mathrm{NG}$ formation is mainly dependent on the non-covalent interactions between ALG, $\mathrm{Ca}^{2+}$ and the encapsulated drugs. The concentration of each component, the cross-linking time, and the $\mathrm{pH}$ to prepare $\mathrm{NG}$ could control the final parameters of $\mathrm{ALG} / \mathrm{Ca}^{2+} \mathrm{NG}$ such as the size, drug encapsulation efficiency, and drug release behavior
(Choukaife et al., 2020). Despite the facile preparation and promising biomedical applications of the self-assembled ALG NG, a comprehensive evaluation of the biocompatibility of ALG is also required for the applications of drug delivery. Most of the ALGs are extracted from algae and bacteria, and meanwhile the preparation of ALG NG may require the assistance of solvent. The impurities, such as endotoxin and residual solvent in ALG NG, could also induce unexpected side effects. Endotoxins are reported to induce immunogenicity and thus compromise the biocompatibility of ALG NG (Lee and Mooney, 2012; Choukaife et al., 2020). The direct self-assembly of purified ALG and drug without the involvement of strong solvent may represent a potent strategy for drug delivery with improved safety profiles.

\section{SELF-ASSEMBLED HYALURONAN (HA) NG}

Besides the CS and ALG, the reports of HA for PS NG preparation are increasing quickly for the past decade. HA, also named as hyaluronic acid and hyaluronate, is a linear and non-sulfated PS composed of repeating disaccharides of D-glucuronic acid and 
$\mathrm{N}$-acetyl-D-glucosamine units (Jeong et al., 2008). HA is highly negatively charged and a major component of the extracellular matrix of mammalian cells (Debele et al., 2016; Miao et al., 2018; Yu et al., 2020). Due to its excellent biocompatibility, low cost, non-immunogenicity, and water-binding properties, HA has been used for dermal filler, joint lubrication, prevention of surgical adhesion, dietary supplements, and eye drops (Valachova et al., 2016; Kim et al., 2017). HA could be specifically recognized by its receptors such as CD44, endocytosed and trafficked to endolysosomes, degraded by hyaluronidase, and then recycled for HA synthesis (Gao et al., 2021). Consequently, the HA-based drug delivery system could realize CD44-targeting as well as hyaluronidase-responsive drug delivery for different cancers. The carboxylic acid, $\mathrm{N}$-acetyl groups, and primary and secondary hydroxyl groups on HA provide chemical modification sites to produce numerous HA derivatives, such as HA-drug conjugates, which have entered the clinical trials (Miao et al., 2018). The carboxyl groups of HA also provide non-covalent cross-linking sites for both $\mathrm{Fe}^{3+}$ and cisplatin to generate NG through selfassembly (Jeong et al., 2008; Isayeva et al., 2010; Tian et al., 2016; Zhang and Tung, 2017).

Currently, the most used non-covalent cross-linker for HA NG is the cisplatin. Cisplatin is a first-line chemotherapeutics for multiple cancers. After the cellular uptake, cisplatin could be aquated and thus get activated in the cytosol (Johnstone et al., 2016). The anticancer activity of cisplatin relies on the DNA cross-linking mediated by the platinum coordination. Similar to its coordination with DNA, cisplatin could also coordinate with the carboxyl groups of various polymers, e.g., HA (Uchino et al., 2005; Jeong et al., 2008; Zhang et al., 2017). The early preparation of HA and cisplatin (HA/Cis) NG employs the silver nitrate to induce the cisplatin aquation, which facilitates its coordination with HA (Cai et al., 2008; Jeong et al., 2008). A simple heating of HA and cisplatin was then found to be capable of accelerating the self-assembly process and thus efficiently tuning the size of the resultant NG (Li and Howell, 2010). The cisplatin in HA/Cis NG not only functions as a cross-linker during the NG formation but also acts as a drug on its release. The existence of chloride reverses the coordination between cisplatin and HA, which could realize a controlled release of cisplatin and thus reduced its notorious side effects in cancer chemotherapy (Cai et al., 2010; Ishiguro et al., 2016). Interestingly, HA and cisplatin could also be co-assembled with a second drug to form multidrug NG (Figure 1C), thus easily realizing the combination therapy especially for cancer (Zhang and Tung, 2017). The cationic-aromatic drugs (CA drugs) containing aromatic ring and positive charges, such as chemotherapeutics (Zhang et al., 2017, 2018; Yu et al., 2020; Ma et al., 2021), kinase inhibitors (Zhang and Tung, 2017), and photosensitizers/dyes (Zhang and Tung, 2018a), could be easily self-assembled into the HA/Cis NG. The positive charges of CA drugs are believed to interact with HA through electrostatic interaction while the aromatic ring contributes to the hydrophobic interaction and $\pi-\pi$ stacking of drugs, which collectively facilitates the encapsulation of CA drug into the NG. Typically, heating of the mixture of HA, cisplatin, and CA drugs could produce a multidrug HA NG. The heating time, ratio of cisplatin to $\mathrm{CA}$ drug, and the CA drug identity were found to determine the final physicochemical properties of the NG such as size, the encapsulation efficiency of drug, and the release speed of drug (Zhang and Tung, 2017). Currently, doxorubicin, gefitinib, dasatinib, toluidine blue, etc. have been successfully co-assembled into the HA/Cis NG (Gao et al., 2021). The HA/Cis NG with doxorubicin encapsulation demonstrated a $\mathrm{pH}$ - and GSH-responsive release behavior, which was able to help combat the cancer drug resistance (Zhang and Tung, 2018b; Ma et al., 2021). Generally, the HA/Cis NG could efficiently realize a CD44-targeted delivery for cancers such as cisplatin monotherapy and cisplatin-based combination therapy. However, cisplatin as a cross-linker in HA/Cis NG inevitably limits its application to malignant diseases. After the self-assembly of HA, cisplatin and CA drugs, a simple dialysis in phosphate-buffered saline (PBS) could remove the cisplatin while keep the CA drugs retained in the NG (Zhang et al., 2017). Subsequently, cisplatin could also be a removable crosslinker to help construct self-assembled HA NG loaded with a single drug, which potentially extends the application of HA NG to other non-cancerous diseases. Different CA drugs could be self-assembled into the HA/Cis NG, and it is still a challenge to realize the macromolecular drug delivery using the native $\mathrm{HA}$.

\section{CONCLUSIONS AND PERSPECTIVES}

The ionic gelation mediated by electrostatic interaction and metal coordination has provided a very facile and biocompatible option to construct the drug-loaded NG through the self-assembly of natural PS such as CS, ALG, and HA. Other than the three PS discussed earlier, ionic gelation of gellan gum and pectin with cationic ions were also used; however, the applications of these PS NG were relatively rare (Racovita et al., 2009; Pedroso-Santana and Fleitas-Salazar, 2020). Future efforts can also focus on exploring different PS NG using the self-assembling strategy for drug delivery. A full understanding of the mechanism between the self-assembly of native PS, ionic, or metal crosslinkers as well as the drug of interest will help quickly obtain the drug-loaded NG with desired properties. For example, the gelation of CS by the TPP was occurred instantly, which made the mechanism study of the NG formation more challenging (Desai, 2016). Adding sodium chloride during the CS/TPP NG preparation could screen the charges and slow the gelation process, which also helped narrow the NG distribution with improved stability (Huang and Lapitsky, 2011, 2017). In terms of the application of drug delivery, ideally, the drug needs to be specifically delivered to the site of action while reducing its non-specific toxicity as much as possible, which requires the nanocarriers to overcome different barriers in the body (Polo et al., 2017; Mitchell et al., 2021). The self-assembled PS NG provide multiple benefits to overcome a part of the above barriers. However, disadvantages also existed for each specific PS NG in the drug delivery. The hydrophilic HA itself has an antifouling property, and meanwhile, it could mediate a targeted delivery of drugs to the tumor with CD44 overexpression (Xia et al., 2019; Lee et al., 2020). However, most of the HA NP 
are retained in the endolysosomes following the trafficking of HA, which largely prevents the delivered drugs to access their molecular targets and subsequently limits the final therapeutic efficacy. To increase the cytosolic drug delivery of HA NG, the improvement in their lysosomal escape capability may also be considered. Currently, several applications of PS-based drug delivery are in different phases of clinical trials (Miao et al., 2018). Although most of them are PS-drug conjugates, the successful demonstration of these PS-based formulations in patients strongly supports the promising therapeutic benefits of PS as a drug carrier. For example, the self-assembled HA/Cis NG (HylaPlat) is being evaluated in phase I/II clinical trial for cancers in dogs (Cai et al., 2016). Generally, the self-assembled NG using the native PS that are free of chemical reactions and meanwhile with ease to scale up will have their own advantages for the translational applications. Before their clinical translation, a thorough evaluation of the therapeutic efficacy as well as the comprehensive safety evaluation are highly recommended

\section{REFERENCES}

Abbaszadeh, S., Rashidipour, M., Khosravi, P., Shahryarhesami, S., Ashrafi, B., Kaviani, M., et al. (2020). Biocompatibility, cytotoxicity, antimicrobial and epigenetic effects of novel chitosan-based quercetin nanohydrogel in human cancer cells. Int. J. Nanomed. 15, 5963-5975. doi: 10.2147/IJN.S263013

Anselmo, A. C., Zhang, M., Kumar, S., Vogus, D. R., Menegatti, S., Helgeson, M. E., et al. (2015). Elasticity of nanoparticles influences their blood circulation, phagocytosis, endocytosis, and targeting. ACS Nano 9, 3169-3177. doi: 10.1021/acsnano.5b00147

Barclay, T. G., Day, C. M., Petrovsky, N., and Garg, S. (2019). Review of polysaccharide particle-based functional drug delivery. Carbohydr. Polym. 221, 94-112. doi: 10.1016/j.carbpol.2019.05.067

Bazban-Shotorbani, S., Dashtimoghadam, E., Karkhaneh, A., Hasani-Sadrabadi, M. M., and Jacob, K. I. (2016). Microfluidic directed synthesis of alginate nanogels with tunable pore size for efficient protein delivery. Langmuir 32, 4996-5003. doi: 10.1021/acs.langmuir.5b04645

Bekale, L., Agudelo, D., and Tajmir-Riahi, H. A. (2015). Effect of polymer molecular weight on chitosan-protein interaction. Colloids Surf. B. Biointerfaces 125, 309-317. doi: 10.1016/j.colsurfb.2014.11.037

Bhattacharya, D. S., Svechkarev, D., Souchek, J. J., Hill, T. K., Taylor, M. A., Natarajan, A., et al. (2017). Impact of structurally modifying hyaluronic acid on CD44 interaction. J. Mater. Chem. B 5, 8183-8192. doi: 10.1039/C7TB01895A

Bowman, K., and Leong, K. W. (2006). Chitosan nanoparticles for oral drug and gene delivery. Int. J. Nanomed 1, 117-128. doi: 10.2147/nano.2006.1.2.117

Brus, J., Urbanova, M., Czernek, J., Pavelkova, M., Kubova, K., Vyslouzil, J., et al. (2017). Structure and dynamics of alginate gels cross-linked by polyvalent ions probed via solid state NMR spectroscopy. Biomacromolecules 18, 2478-2488. doi: 10.1021/acs.biomac.7b00627

Cai, S., Xie, Y., Bagby, T. R., Cohen, M. S., and Forrest, M. L. (2008). Intralymphatic chemotherapy using a hyaluronan-cisplatin conjugate. J. Surg. Res. 147, 247252. doi: 10.1016/j.jss.2008.02.048

Cai, S., Xie, Y., Davies, N. M., Cohen, M. S., and Forrest, M. L. (2010). Pharmacokinetics and disposition of a localized lymphatic polymeric hyaluronan conjugate of cisplatin in rodents. J. Pharm. Sci. 99, 2664-2671. doi: $10.1002 /$ jps.22016

Cai, S., Zhang, T., Forrest, W. C., Yang, Q., Groer, C., Mohr, E., et al. (2016). Phase I-II clinical trial of hyaluronan-cisplatin nanoconjugate in dogs with naturally occurring malignant tumors. Am. J. Vet. Res. 77, 1005-1016. doi: 10.2460/ajvr.77.9.1005

Cai, Y. H., and Lapitsky, Y. (2020). Biomolecular uptake effects on chitosan/tripolyphosphate micro- and nanoparticle stability. Colloids Surf. B. 193:111081. doi: 10.1016/j.colsurfb.2020.111081 using the clinically relevant animal models according to the independent application.

\section{AUTHOR CONTRIBUTIONS}

WZ and HW conceived and drafted the manuscript including the figures. $\mathrm{HD}$ and $\mathrm{MG}$ participated in reviewing the literature, writing, and discussions. All authors approved the submitted version.

\section{FUNDING}

This work was partly supported by the Beijing Nova Program (Z201100006820110) of Beijing Municipal Science \& Technology Commission, the CAMS Innovation Fund for Medical Science (CIFMS 2019-I2M-1-004), the Fundamental Research Funds for the Central Universities (3332019064), and the grant from the State Key Laboratory Special Fund (2060204).

Calvo, P., Remunan-Lopez, C., Vila-Jato, J. L., and Alonso, M. (1997) Novel hydrophilic chitosan-polyethylene oxide nanoparticles as protein carriers. J. Appl. Polym. Sci. 63, 125-132. doi: 10.1002/(SICI)10974628(19970103)63:1<125::AID-APP13>3.0.CO;2-4

Cheung, R. C. F., Ng, T. B., Wong, J. H., and Chan, W. Y. (2015). Chitosan: an update on potential biomedical and pharmaceutical applications. Mar. Drugs 13, 5156-5186. doi: 10.3390/md13085156

Choukaife, H., Doolaanea, A. A., and Alfatama, M. (2020). Alginate nanoformulation: influence of process and selected variables. Pharmaceuticals 13:335. doi: 10.3390/ph13110335

Corbo, C., Molinaro, R., Parodi, A., Toledano Furman, N. E., Salvatore, F., and Tasciotti, E. (2016). The impact of nanoparticle protein corona on cytotoxicity, immunotoxicity and target drug delivery. Nanomedicine 11, 81-100. doi: 10.2217/nnm.15.188

Cuggino, J. C., Blanco, E. R. O., Gugliotta, L. M., Igarzabal, C. I. A., and Calderon, M. (2019). Crossing biological barriers with nanogels to improve drug delivery performance. J. Controlled Release 307, 221-246. doi: 10.1016/j.jconrel.2019.06.005

Debele, T. A., Mekuria, S. L., and Tsai, H. C. (2016). Polysaccharide based nanogels in the drug delivery system: application as the carrier of pharmaceutical agents. Mater. Sci. Eng. C. 68, 964-981. doi: 10.1016/j.msec.2016. 05.121

Degors, I. M. S., Wang, C., Rehman, Z. U., and Zuhorn, I. S. (2019). Carriers break barriers in drug delivery: endocytosis and endosomal escape of gene delivery vectors. Acc. Chem. Res. 52, 1750-1760. doi: 10.1021/acs.accounts. $9 \mathrm{~b} 00177$

Desai, K. G. H. (2016). Chitosan nanoparticles prepared by ionotropic gelation: an overview of recent advances. Crit. Rev. Ther. Drug Carrier Syst. 33, 107-158. doi: 10.1615/CritRevTherDrugCarrierSyst.2016014850

Fan, W., Yan, W., Xu, Z., and Ni, H. (2012). Formation mechanism of monodisperse, low molecular weight chitosan nanoparticles by ionic gelation technique. Colloids Surf. B. Biointerfaces 90, 21-27. doi: 10.1016/j.colsurfb.2011.09.042

Fischetti, T., Celikkin, N., Contessi Negrini, N., Fare, S., and Swieszkowski, W. (2020). Tripolyphosphate-crosslinked chitosan/gelatin biocomposite ink for 3D printing of uniaxial scaffolds. Front. Bioeng. Biotechnol. 8:400. doi: $10.3389 /$ fbioe. 2020.00400

Gao, M. H., Deng, H., and Zhang, W. Q. (2021). Hyaluronan-based multifunctional nano-carriers for combination cancer therapy. Curr. Top. Med. Chem. 21, 126-139. doi: 10.2174/1568026620666200922113846

Giammanco, G. E., Sosnofsky, C. T., and Ostrowski, A. D. (2015). Light-responsive Iron(III)-polysaccharide coordination hydrogels for controlled delivery. ACS Appl. Mater. Interfaces 7, 3068-3076. doi: 10.1021/am506772x 
Grant, G. T., Morris, E. R., Rees, D. A., Smith, P. J., and Thom, D. (1973). Biological interactions between polysaccharides and divalent cations: the egg-box model. FEBS Lett. 32, 195-198. doi: 10.1016/0014-5793(73)80770-7

Guo, P., Liu, D. X., Subramanyam, K., Wang, B. R., Yang, J., Huang, J., et al. (2018). Nanoparticle elasticity directs tumor uptake. Nat. Commun. 9:130. doi: 10.1038/s41467-017-02588-9

Hartshorn, C. M., Bradbury, M. S., Lanza, G. M., Nel, A. E., Rao, J. H., Wang, A. Z., et al. (2018). Nanotechnology strategies to advance outcomes in clinical cancer care. ACS Nano 12, 24-43. doi: 10.1021/acsnano.7b05108

Hendrickson, G. R., and Lyon, L. A. (2010). Microgel translocation through pores under confinement. Angew. Chem. Int. Ed. 49, 2193-2197. doi: $10.1002 /$ anie. 200906606

Hu, C., Lu, W., Mata, A., Nishinari, K., and Fang, Y. (2021). Ions-induced gelation of alginate: mechanisms and applications. Int. J. Biol. Macromol. 177, 578-588. doi: 10.1016/j.ijbiomac.2021.02.086

Huang, Y., and Lapitsky, Y. (2011). Monovalent salt enhances colloidal stability during the formation of chitosan/tripolyphosphate microgels. Langmuir 27, 10392-10399. doi: 10.1021/la201194a

Huang, Y., and Lapitsky, Y. (2017). On the kinetics of chitosan/tripolyphosphate micro- and nanogel aggregation and their effects on particle polydispersity. J. Colloid Interface Sci. 486, 27-37. doi: 10.1016/j.jcis.2016.09.050

Isayeva, I., Sarkar Das, S., Chang, A., Defoe, J., Luu, H. M., Vorvolakos, K., et al. (2010). pH effect on the synthesis, shear properties, and homogeneity of ironcrosslinked hyaluronic acid-based gel/adhesion barrier. J. Biomed. Mater. Res. B Appl. Biomater. 95, 9-18. doi: 10.1002/jbm.b.31677

Ishiguro, S., Cai, S., Uppalapati, D., Turner, K., Zhang, T., Forrest, W. C., et al. (2016). Intratracheal administration of hyaluronan-cisplatin conjugate nanoparticles significantly attenuates lung cancer growth in mice. Pharm. Res. 33, 2517-2529. doi: 10.1007/s11095-016-1976-3

Jamil, B., Habib, H., Abbasi, S., Nasir, H., Rahman, A., Rehman, A., et al. (2016). Cefazolin loaded chitosan nanoparticles to cure multi drug resistant Gram-negative pathogens. Carbohydr. Polym. 136, 682-691. doi: 10.1016/j.carbpol.2015.09.078

Jeong, Y. I., Kim, S. T., Jin, S. G., Ryu, H. H., Jin, Y. H., Jung, T. Y., et al. (2008). Cisplatin-incorporated hyaluronic acid nanoparticles based on ion-complex formation. J. Pharm. Sci. 97, 1268-1276. doi: 10.1002/jps.21103

Johnstone, T. C., Suntharalingam, K., and Lippard, S. J. (2016). The next generation of platinum drugs: targeted $\mathrm{Pt}(\mathrm{II})$ agents, nanoparticle delivery, and $\mathrm{Pt}(\mathrm{IV})$ prodrugs. Chem. Rev. 116, 3436-3486. doi: 10.1021/acs.chemrev.5b00597

Katas, H., and Alpar, H. O. (2006). Development and characterisation of chitosan nanoparticles for siRNA delivery. J. Controlled Release 115, 216-225. doi: 10.1016/j.jconrel.2006.07.021

Kim, H., Jeong, H., Han, S., Beack, S., Hwang, B. W., Shin, M., et al. (2017). Hyaluronate and its derivatives for customized biomedical applications. Biomaterials 123, 155-171. doi: 10.1016/j.biomaterials.2017.01.029

Kim, H., Park, Y., Stevens, M. M., Kwon, W., and Hahn, S. K. (2019). Multifunctional hyaluronate-nanoparticle hybrid systems for diagnostic, therapeutic and theranostic applications. J. Controlled Release 303, 55-66. doi: 10.1016/j.jconrel.2019.04.003

Kobayashi, H., Watanabe, R., and Choyke, P. L. (2014). Improving conventional enhanced permeability and retention (EPR) effects; What is the appropriate target? Theranostics 4, 81-89. doi: 10.7150/thno.7193

Lapitsky, Y. (2014). Ionically crosslinked polyelectrolyte nanocarriers: recent advances and open problems. Curr. Opin. Colloid Interface Sci. 19, 122-130. doi: 10.1016/j.cocis.2014.03.014

Lee, K. Y., and Mooney, D. J. (2012). Alginate: properties and biomedical applications. Prog. Polym. Sci. 37, 106-126. doi: 10.1016/j.progpolymsci.2011.06.003

Lee, S. Y., Kang, M. S., Jeong, W. Y., Han, D. W., and Kim, K. S. (2020). Hyaluronic acid-based theranostic nanomedicines for targeted cancer therapy. Cancers 12:940. doi: $10.3390 /$ cancers 12040940

Li, S.-D., and Howell, S. B. (2010). CD44-targeted microparticles for delivery of cisplatin to peritoneal metastases. Mol. pharmaceutics 7, 280-290. doi: $10.1021 / \mathrm{mp} 900242 \mathrm{f}$

Li, Z., Xiao, C., Yong, T. Y., Li, Z. F., Gan, L., and Yang, X. L. (2020). Influence of nanomedicine mechanical properties on tumor targeting delivery. Chem. Soc. Rev. 49, 2273-2290. doi: 10.1039/C9CS00575G
Liu, Z. H., Jiao, Y. P., Wang, Y. F., Zhou, C. R., and Zhang, Z. Y. (2008). Polysaccharides-based nanoparticles as drug delivery systems. Adv. Drug Delivery Rev. 60, 1650-1662. doi: 10.1016/j.addr.2008.09.001

Ma, W., Chen, Q., Xu, W., Yu, M., Yang, Y., Zou, B., et al. (2021). Self-targeting visualizable hyaluronate nanogel for synchronized intracellular release of doxorubicin and cisplatin in combating multidrug-resistant breast cancer. Nano Res. 14, 846-857. doi: 10.1007/s12274-020-3124-y

Mauri, E., Perale, G., and Rossi, F. (2018). Nanogel Functionalization: a versatile approach to meet the challenges of drug and gene delivery. ACS Appl. Nano Mater. 1, 6525-6541. doi: 10.1021/acsanm.8b01686

Miao, T. X., Wang, J. Q., Zeng, Y., Liu, G., and Chen, X. Y. (2018). Polysaccharide-based controlled release systems for therapeutics delivery and tissue engineering: from bench to bedside. Adv. Sci. 5:1700513. doi: 10.1002/advs.201700513

Mitchell, M. J., Billingsley, M. M., Haley, R. M., Wechsler, M. E., Peppas, N. A., and Langer, R. (2021). Engineering precision nanoparticles for drug delivery. Nat. Rev. Drug Discov. 20, 101-124. doi: 10.1038/s41573-020-0090-8

Moraru, C., Mincea, M., Menghiu, G., and Ostafe, V. (2020). Understanding the factors influencing chitosan-based nanoparticles-protein corona interaction and drug delivery applications. Molecules 25:4758. doi: 10.3390/molecules 25204758

Morin-Crini, N., Lichtfouse, E., Torri, G., and Crini, G. (2019). Applications of chitosan in food, pharmaceuticals, medicine, cosmetics, agriculture, textiles, pulp and paper, biotechnology, and environmental chemistry. Environ. Chem. Lett. 17, 1667-1692. doi: 10.1007/s10311-019-00904-x

Nasti, A., Zaki, N. M., De Leonardis, P., Ungphaiboon, S., Sansongsak, P., Rimoli, M. G., et al. (2009). Chitosan/TPP and chitosan/TPP-hyaluronic acid nanoparticles: systematic optimisation of the preparative process and preliminary biological evaluation. Pharm. Res. 26, 1918-1930. doi: 10.1007/s11095-009-9908-0

Nogueira, D. R., Tavano, L., Mitjans, M., Perez, L., Infante, M. R., and Vinardell, M. P. (2013). In vitro antitumor activity of methotrexate via $\mathrm{pH}$-sensitive chitosan nanoparticles. Biomaterials 34, 2758-2772. doi: 10.1016/j.biomaterials.2013.01.005

Ojeda-Hernandez, D. D., Canales-Aguirre, A. A., Matias-Guiu, J., Gomez-Pinedo, U., and Mateos-Diaz, J. C. (2020). Potential of chitosan and its derivatives for biomedical applications in the central nervous system. Front. Bioeng. Biotechnol. 8:389. doi: 10.3389/fbioe.2020.00389

Pedroso-Santana, S., and Fleitas-Salazar, N. (2020). Ionotropic gelation method in the synthesis of nanoparticles/microparticles for biomedical purposes. Polym. Int. 69, 443-447. doi: 10.1002/pi.5970

Polo, E., Collado, M., Pelaz, B., and Del Pino, P. (2017). Advances toward more efficient targeted delivery of nanoparticles in vivo: understanding interactions between nanoparticles and cells. ACS Nano 11, 2397-2402. doi: $10.1021 /$ acsnano.7b01197

Qu, B., and Luo, Y. C. (2020). Chitosan-based hydrogel beads: preparations, modifications and applications in food and agriculture sectors-a review. Int. J. Biol. Macromol. 152, 437-448. doi: 10.1016/j.ijbiomac.2020.02.240

Racovita, S., Vasiliu, S., Popa, M., and Luca, C. (2009). Polysaccharides based on micro- and nanoparticles obtained by ionic gelation and their applications as drug delivery systems. Revue Roumaine De Chimie 54, 709-718.

Renu, S., and Renukaradhya, G. J. (2020). Chitosan nanoparticle based mucosal vaccines delivered against infectious diseases of poultry and pigs. Front. Bioeng. Biotechnol. 8:558349. doi: 10.3389/fbioe.2020.558349

Richard, I., Thibault, M., De Crescenzo, G., Buschmann, M. D., and Lavertu, M. (2013). Ionization behavior of chitosan and chitosan-DNA polyplexes indicate that chitosan has a similar capability to induce a proton-sponge effect as PEI. Biomacromolecules 14, 1732-1740. doi: 10.1021/bm4000713

Russo, R., Malinconico, M., and Santagata, G. (2007). Effect of cross-linking with calcium ions on the physical properties of alginate films. Biomacromolecules 8 , 3193-3197. doi: 10.1021/bm700565h

Severino, P., Da Silva, C. F., Andrade, L. N., De Lima Oliveira, D., Campos, J., and Souto, E. B. (2019). Alginate nanoparticles for drug delivery and targeting. Curr. Pharm. Des. 25, 1312-1334. doi: 10.2174/1381612825666190425163424

Soni, K. S., Desale, S. S., and Bronich, T. K. (2016). Nanogels: an overview of properties, biomedical applications and obstacles to clinical translation. J. Controlled Release 240, 109-126. doi: 10.1016/j.jconrel.2015.11.009 
Spadea, A., De La Rosa, J. M. R., Tirella, A., Ashford, M. B., Williams, K. J., Stratford, I. J., et al. (2019). Evaluating the efficiency of hyaluronic acid for tumor targeting via CD44. Mol. Pharm. 16, 2481-2493. doi: 10.1021/acs.molpharmaceut.9b00083

Sreekumar, S., Goycoolea, F. M., Moerschbacher, B. M., and Rivera-Rodriguez, G. R. (2018). Parameters influencing the size of chitosan-TPP nano- and microparticles. Sci. Rep. 8:4695. doi: 10.1038/s41598-018-23064-4

Suhail, M., Rosenholm, J. M., Minhas, M. U., Badshah, S. F., Naeem, A., Khan, K. U., et al. (2019). Nanogels as drug-delivery systems: a comprehensive overview. Ther. Deliv. 10, 697-717. doi: 10.4155/tde-2019-0010

Swierczewska, M., Han, H. S., Kim, K., Park, J. H., and Lee, S. (2016). Polysaccharide-based nanoparticles for theranostic nanomedicine. Adv. Drug Deliv. Rev. 99, 70-84. doi: 10.1016/j.addr.2015.11.015

Tian, Y., Guo, R., Wang, Y., and Yang, W. (2016). Coordination-induced assembly of intelligent polysaccharide-based phototherapeutic nanoparticles for cancer treatment. Adv. Healthc. Mater. 5, 3099-3104. doi: 10.1002/adhm.201600877

Uchino, H., Matsumura, Y., Negishi, T., Koizumi, F., Hayashi, T., Honda, T., et al. (2005). Cisplatin-incorporating polymeric micelles (NC-6004) can reduce nephrotoxicity and neurotoxicity of cisplatin in rats. Br. J. Cancer 93, 678-687. doi: 10.1038/sj.bjc.6602772

Valachova, K., Volpi, N., Stern, R., and Soltes, L. (2016). Hyaluronan in medical practice. Curr. Med. Chem. 23, 3607-3617. doi: 10.2174/0929867323666160824162133

Xia, Y. Q., Adibnia, V., Shan, C. C., Huang, R. L., Qi, W., He, Z. M., et al. (2019). Synergy between zwitterionic polymers and hyaluronic acid enhances antifouling performance. Langmuir 35, 15535-15542. doi: 10.1021/acs.langmuir.9b01876

Xu, Y., Asghar, S., Gao, S., Chen, Z., Huang, L., Yin, L., et al. (2017). Polysaccharidebased nanoparticles for co-loading mitoxantrone and verapamil to overcome multidrug resistance in breast tumor. Int. J. Nanomed. 12, 7337-7350. doi: $10.2147 /$ IJN.S145620

$\mathrm{Xu}, \mathrm{Y}$., and Du, Y. (2003). Effect of molecular structure of chitosan on protein delivery properties of chitosan nanoparticles. Int. J. Pharm. 250, 215-226. doi: 10.1016/S0378-5173(02)00548-3

Xue, Y., Xia, X., Yu, B., Luo, X., Cai, N., Long, S., et al. (2015). A green and facile method for the preparation of a $\mathrm{pH}$-responsive alginate nanogel for subcellular delivery of doxorubicin. RSC Adv. 5, 73416-73423. doi: 10.1039/C5RA13313K
Yu, T., Li, Y., Gu, X., and Li, Q. (2020). Development of a hyaluronic acidbased nanocarrier incorporating doxorubicin and cisplatin as a $\mathrm{pH}$-sensitive and CD44-targeted anti-breast cancer drug delivery system. Front. Pharmacol. 11:532457. doi: 10.3389/fphar.2020.532457

Zhang, W., and Tung, C. H. (2018a). Real-time visualization of lysosome destruction using a photosensitive toluidine blue nanogel. Chem. Eur. J. 24, 2089-2093. doi: 10.1002/chem.201705697

Zhang, W., Zhang, Z., and Tung, C.-H. (2017). Beyond chemotherapeutics: cisplatin as a temporary buckle to fabricate drug-loaded nanogels. Chem. Commun. 53, 779-782. doi: 10.1039/C6CC08230K

Zhang, W. Q., Han, B., Lai, X. N., Xiao, C., Xu, S. L., Meng, X. H., et al. (2020). Stiffness of cationized gelatin nanoparticles is a key factor determining RNAi efficiency in myeloid leukemia cells. Chem. Commun. 56, 1255-1258. doi: 10.1039/C9CC09068A

Zhang, W. Q., and Tung, C. H. (2017). Cisplatin cross-linked multifunctional nanodrugplexes for combination therapy. ACS Appl. Mater. Interfaces 9, 8547-8555. doi: 10.1021/acsami. 6b16500

Zhang, W. Q., and Tung, C. H. (2018b). Redox-responsive cisplatin nanogels for anticancer drug delivery. Chem. Commun. 54, 8367-8370. doi: 10.1039/C8CC01795F

Zhang, Y., Wang, F., Li, M., Yu, Z., Qi, R., Ding, J., et al. (2018). Selfstabilized hyaluronate nanogel for intracellular codelivery of doxorubicin and cisplatin to osteosarcoma. Adv. Sci. 5:1700821. doi: 10.1002/advs.2017 00821

Conflict of Interest: The authors declare that the research was conducted in the absence of any commercial or financial relationships that could be construed as a potential conflict of interest.

Copyright (c) 2021 Wang, Deng, Gao and Zhang. This is an open-access article distributed under the terms of the Creative Commons Attribution License (CC BY). The use, distribution or reproduction in other forums is permitted, provided the original author(s) and the copyright owner(s) are credited and that the original publication in this journal is cited, in accordance with accepted academic practice. No use, distribution or reproduction is permitted which does not comply with these terms. 